\title{
Solution of Modified Equations of Emden-Type by Differential Transform Method
}

\author{
Supriya Mukherjee ${ }^{1}$, Banamali Roy ${ }^{2}$, Pratik Kumar Chatterjee ${ }^{3}$ \\ ${ }^{1}$ Department of Mathematics Swami Vivekananda Institute of Science \& Technology, \\ South Gobindapur, P.S-Sonarpur, Kolkata, West Bengal, India \\ ${ }^{2}$ Department of Mathematics Bangabasi Evening College, 19 Rajkumar Chakraborty Sarani, \\ Kolkata, West Bengal, India \\ ${ }^{3}$ Department of Computer Science and Engineering Swami Vivekananda Institute of Science \& Technology, \\ South Gobindapur, P.S-Sonarpur, Kolkat, West Bengal, India \\ E-mail: supriya_ju@yahoo.com,banamaliroy@yahoo.co.in,pratik_kumar_chatterjee@yahoo.in \\ Received February 2, 2011; revised March 24, 2011; accepted April 6, 2011
}

\begin{abstract}
In this paper the Modified Equations of Emden type (MEE), $\ddot{x}+\alpha x \dot{x}+\beta x^{3}=0$ is solved numerically by the differential transform method. This technique doesn't require any discretization, linearization or small perturbations and therefore it reduces significantly the numerical computation. The current results of this paper are in excellent agreement with those provided by Chandrasekar et al. [1] and thereby illustrate the reliability and the performance of the differential transform method. We have also compared the results with the classical Runge-Kutta 4 (RK4) Method.
\end{abstract}

Keywords: Modified Equations of Emden Type, Differential Transforms Method, Runge-Kutta 4 (RK4) Method

\section{Introduction}

The modified equation of Emden type (MEE), also called the modified Painleve-Ince equation,

$$
\ddot{x}+\alpha x \dot{x}+\beta x^{3}=0
$$

where over dot denotes differentiation with respect to time and $\alpha$ and $\beta$ are arbitrary parameters, have received attention from both mathematicians and physicists for more than a century [2-6]. The above differential equation appears in a number of mathematical problems such as univalued functions defined by second order differential equations [7] and the Riccati equation [8]. Physicists have found this equation in the study of equilibrium configurations of a spherical gas cloud acting under the mutual attraction of its molecules and subject to the laws of thermodynamics [9-12], in spherically symmetric expansion or collapse of a relativistic gravitating mass [13] and in the modeling of the fusion of pellets [14]. The invariance and the integrability of this equation have been a subject of study for the past two decades by a number of authors [15-26]. This equation have been found to possess an explicit general solution for the following parametric choices,

$$
\begin{aligned}
& \alpha=0, \\
& \beta=0, \\
& \beta=\frac{\alpha^{2}}{9} \\
& \beta=-\alpha^{2}
\end{aligned}
$$

However, the general solution of Equation (1) for arbitrary values of $\alpha$ and $\beta$ was explored for the first time by Chandrasekhar et al. [1]. They have constructed the time-independent Hamiltonians from the time-independent integrals of Equation (1) and by the suitable use of canonical transformations, have converted these Hamiltonians to their standard forms. The general solutions are then obtained by integrating these new Hamiltonians. We present here a humble effort to arrive at the same by the Differential Transform Method [DTM].

The concept of differential transform was first introduced by Zhou [27] in solving linear and nonlinear initial value problems in electrical circuit analysis. The traditional Taylor series method takes a long time for compu- 
tation of higher order derivatives. Instead, DTM is an iterative procedure for obtaining analytic Taylor series solution of differential equations and is much easier. In our previous work we have seen that the DTM provides the solution of the Duffing-Van der Pol oscillator equation in a rapidly convergent series [28] and that, it is in good agreement with the solution obtained by Chandrasekar et al. [29].

\section{The Modified Emden-Type Equations}

As already mentioned, the modified equation of Emden type cannot be integrated straightforwardly for arbitrary values of $\alpha$ and $\beta$. The solution of MEE for the particular choice of parameters given by (2a) and ( $2 b)$ can be obtained by simple integration and for the choice (2c), the equation is linearizable to a free particle equation. In the fourth case the general solution can be expressed in terms of the Weierstrass elliptic function [2-6,15-26,30]. It has also been noted that the MEE possess the Painleve property for certain values of $r=\left(\frac{\alpha}{4 \beta}\right)\left(\alpha \pm \sqrt{\alpha^{2}-8 \beta}\right)$ [19, 20,22].

In [1] the authors have identified the first integrals of Equation (1) separately for each of the three ranges 1) $\alpha^{2}=8 \beta$, 2) $\alpha^{2}>8 \beta$, and 3) $\alpha^{2}<8 \beta$. The Hamiltonians are obtained from these integrals and are given by

$$
H=\left\{\begin{array}{cc}
\log \left(\frac{-4 \alpha}{p}\right)-\frac{\alpha}{4} p x^{2} & \alpha^{2}=8 \beta \\
\frac{r-1}{r-2} p^{\frac{r-2}{r-1}}-\frac{r-1}{2 r} \alpha x^{2} p & \alpha^{2}>8 \beta \\
\frac{1}{2} \log \left[x^{4} \sec ^{2}\left(\frac{\omega}{4} x^{2} p\right)\right]-\frac{\alpha}{4} x^{2} p & \alpha^{2}<8 \beta
\end{array}\right.
$$

For the case $\alpha^{2}=8 \beta$ the Hamiltonian $H=\log \left(\frac{-4 \alpha}{p}\right)-\frac{\alpha}{4} p x^{2}$ reduces to the standard form

$$
H=\frac{1}{2} p^{2}+\log \left(\frac{32}{U^{2}}\right),
$$

under the canonical transformation

$$
x=\frac{4 P}{\alpha U}, \quad p=-\frac{\alpha U^{2}}{8} .
$$

The general solution thus obtained by integrating the new Hamiltonian (4) and by using the canonical equations $\dot{U}=P$ and $\dot{P}=\frac{2}{U}$ is given by,

$$
x(t)=\frac{8}{i \alpha} \operatorname{erf}^{-1}(z) \exp \left[\frac{1}{2}\left(E+2\left[\operatorname{erf}^{-1}(z)\right]^{2}\right)\right]
$$

where, $z=\frac{\left\{2\left(t_{0}+i t\right) \exp [E / 2]\right\}}{\sqrt{\pi}}, \quad E=\frac{1}{2} \dot{U}^{2}-2 \log U$, $t_{0}$ is an arbitrary constant of integration and erf is the error function [31].

In our present work we have solved the modified equation of Emden type by the Differential transform method and we have compared the results with Equation (6) [1]. We have also compared the results with those obtained by Runge-Kutta 4 Method.

\section{The Differential Transform Method}

Differential transform of a function $f(x)$ is defined as follows

$$
F(k)=\frac{1}{k !}\left[\frac{\mathrm{d}^{k} f(x)}{\mathrm{d} x^{k}}\right]_{x=0} .
$$

In (7), $f(x)$ is the original function and $F(k)$ is the transformed function. The Taylor series expansion of the function $f(x)$ about a point $x=0$ is given as

$$
f(x)=\sum_{k=0}^{\infty} \frac{x^{k}}{k !}\left[\frac{\mathrm{d}^{k} f(x)}{\mathrm{d} x^{k}}\right]_{x=0} .
$$

$$
\begin{gathered}
\text { Replacing } \frac{1}{k !}\left[\frac{\mathrm{d}^{k} f(x)}{\mathrm{d} x^{k}}\right]_{x=0} \text { by } F(k), \text { we have } \\
f(x)=\sum_{k=0}^{\infty} x^{k} F(x)
\end{gathered}
$$

which may be defined as the inverse differential transform.

From (7) and (8) it is easy to obtain the following mathematical operations:

1) If $f(x)=g(x) \pm h(x)$, then $F(k)=G(k) \pm H(k)$

2) If $f(x)=c g(x)$, then $F(k)=c G(k)$, where $c$ is a constant.

3) If then $f(x)=\frac{d^{n} g(x)}{d x^{n}}$, then

$F(k)=\frac{(k+n) !}{k !} G(k+n)$.

4) If $f(x)=g(x) h(x)$, then

$F(k)=\sum_{l=0}^{k} G(l) H(k-l)$.

5) If $f(x)=x^{n}$, then $F(k)=\delta(k-n)$, where $\delta$ is Kronecker delta.

6) If $f(x)=\int_{0}^{x} g(t) \mathrm{d} t$, then $F(k)=\frac{G(k-1)}{k}$, where $k \geq 1$. 
7) If $f(x)=u(x) v(x) w(x)$, then $F(k)=\sum_{s=0}^{k} \sum_{m=0}^{k-s} U(s) V(m) W(k-s-m)$.

where $F(k), G(k), H(k), U(k), V(k), W(k)$ are the differential transform of the functions $f(x)$, $g(x), h(x), u(x), v(x), w(x)$ respectively.

\section{Solution of the Modified Emden-Type Equations Using Differential Transform Method}

The equation of the modified Emden type is given as

$$
\frac{\mathrm{d}^{2} x}{\mathrm{~d} t^{2}}+\alpha x \frac{\mathrm{d} x}{\mathrm{~d} t}+\beta x^{3}=0
$$

The initial conditions are $x(0)=0$ and $x^{\prime}(0)=1$ (where prime denotes differentiation with respect to time).

Applying Differential Transform (DT) to (9), we have

$$
D T\left[\frac{\mathrm{d}^{2} x}{\mathrm{~d} t^{2}}+\alpha x \frac{\mathrm{d} x}{\mathrm{~d} t}+\beta x^{3}=0\right]
$$

or

$$
D T\left[\frac{\mathrm{d}^{2} x}{\mathrm{~d} t^{2}}\right]+\alpha D T\left[x \frac{\mathrm{d} x}{\mathrm{~d} t}\right]+\beta D T\left[x^{3}\right]=0,
$$

i.e.

$$
\begin{aligned}
& (k+2)(k+1) T(k+2)+\alpha \sum_{l=0}^{k} T(l)(k+1-l) T(k+1-l) \\
& \quad+\beta \sum_{s=0}^{k} \sum_{m=0}^{k-s} T(s) T(m) T(k-s-m)=0 .
\end{aligned}
$$

The inverse differential transform of $\mathrm{T}(\mathrm{k})$ is defined as

$$
x(t)=\sum_{k=0}^{\infty} T(k) t^{k} .
$$

Using the initial conditions $x(0)=0 ; \frac{\mathrm{d} x(0)}{\mathrm{d} t}=1$ we have, $T(0)=0$ and $T(1)=1$.

For $k=0$ in the above equation, we have

$2 T(2)+\alpha T(0) T(1)+\beta T(0) T(0) T(0)=0 \Rightarrow T(2)=0$.

For $k=1$, we have

$$
\begin{aligned}
& 6 T(3)+\alpha[2 T(0) T(2)+T(1) T(1)] \\
& +\beta\left[\sum_{m=0}^{1} T(0) T(m) T(1-m)+T(1) T(0) T(0)\right]=0, \\
& \Rightarrow T(3)=\frac{-\alpha}{6} .
\end{aligned}
$$

For $k=2$, we have

$$
\begin{aligned}
& 12 T(4)+\alpha \sum_{l=0}^{23}(3-l) T(l) T(3-l) \\
& +\beta \sum_{s=0}^{2} \sum_{m=0}^{2-s} T(s) T(m) T(2-s-m)=0 \\
& \Rightarrow T(4)=0 .
\end{aligned}
$$

For $k=3,4,5,6,7$, we have

$$
\begin{gathered}
T(5)=\frac{\alpha^{2}}{30}-\frac{\beta}{20}, \\
T(6)=0, \\
T(7)=\frac{-17 \alpha^{3}}{2520}+\frac{2 \alpha \beta}{105}, \\
T(8)=0, \\
T(9)=\frac{1395 \alpha^{4}}{1020600}-\frac{379 \alpha^{2} \beta}{30240}+\frac{3 \beta^{2}}{1440},
\end{gathered}
$$

and

$$
T(10)=0 \text {. }
$$

For the case $\alpha^{2}=8 \beta$, from Equations (11) to (19) we have,

$$
\begin{aligned}
& T(2)=0, \\
& T(3)=-\frac{\alpha}{6}, \\
& T(4)=0, \\
& T(5)=\frac{\alpha^{2}}{30}-\frac{\beta}{20}=\frac{13 \alpha^{2}}{60} \text {, } \\
& T(6)=0 \text {, } \\
& T(7)=\frac{-17 \alpha^{3}}{2520}+\frac{2 \alpha \beta}{105}=\frac{-231 \alpha^{3}}{52920}, \\
& T(8)=0, \\
& T(9)=\left(\frac{1395}{1020600}-\frac{6025}{9072000}+\frac{3}{92160}\right) \alpha^{4} \text {, } \\
& =\frac{735}{1000000} \alpha^{4} \\
& T(10)=0 . \\
& \Rightarrow x(t)=t-t^{3} \frac{\alpha}{6}+t^{5} \frac{13 \alpha^{2}}{60}-t^{7} \frac{231 \alpha^{3}}{52920} \\
& +t^{9} \frac{735 \alpha^{4}}{1000000}-\cdots \infty
\end{aligned}
$$




\section{Comparison of Results}

The solution plot of the Modified Equations of Emden type using DTM is given in Figure 1 for the parametric choice $\alpha^{2}=8 \beta$ for different values of $\alpha$. The graphical representation of the solution (30) obtained by the DTM in this paper is in good agreement with those obtained by Chandrasekar et al. [1] and thereby illustrate the reliability and the performance of the differential transform method. Figure 2 gives us a comparison of the solution for MEE obtained by DTM with the solution obtained by classical Runge-Kutta 4 Method. Table 1 gives the estimate of absolute error between the DTM-solutions with RK4 solutions. It is clear from Figures 1, 2 and Table 1, that the solution obtained by DTM is a better approximation to the exact solution (as obtained in [1]) than the classical RK4 method. Therefore, the DTM is a very efficient and accurate method that can be used to provide analytical solution for nonlinear differential equ-

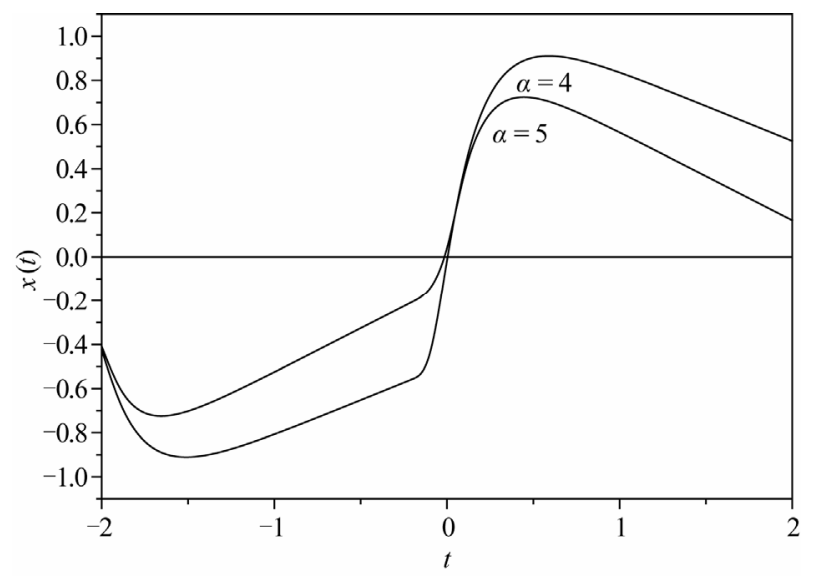

Figure 1. Plot of solution (30) of Modified Equations of Emden type for the case $\alpha^{2}=8 \beta$ taking $\alpha=4$ and $\alpha=5$.

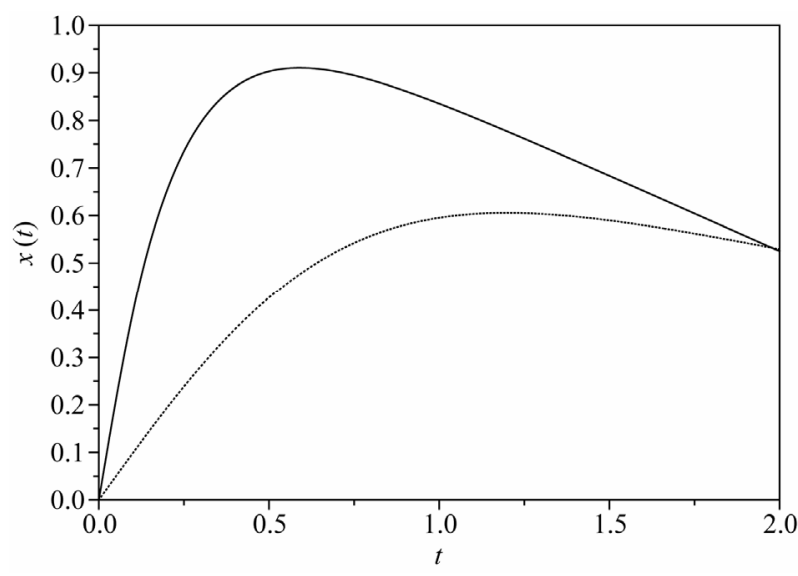

Figure 2. Plot of solution of Modified Equations of Emden type for the case $\alpha^{2}=8 \beta$ taking $\alpha=4$ using DTM [Solid line] and RK4 method [Dotted line].
Table 1. Comparison of the DTM- solutions with RK4 solutions and calculation of Absolute error.

\begin{tabular}{cccc}
\hline Time & RK4 solution & DTM solution $x(t)$ & Absolute error \\
\hline 0 & 0 & 0 & 0 \\
0.1 & 0.09934 & 0.42503 & 0.32569 \\
0.2 & 0.1948 & 0.67042 & 0.47562 \\
0.3 & 0.28299 & 0.80679 & 0.5238 \\
0.4 & 0.36135 & 0.87702 & 0.51567 \\
0.5 & 0.42833 & 0.90712 & 0.47879 \\
0.6 & 0.48334 & 0.91287 & 0.42953 \\
0.7 & 0.52667 & 0.90384 & 0.37717 \\
0.8 & 0.55915 & 0.88584 & 0.32669 \\
0.9 & 0.58201 & 0.8624 & 0.28039 \\
1 & 0.59662 & 0.83567 & 0.23905 \\
1.1 & 0.60436 & 0.80693 & 0.20257 \\
1.2 & 0.60652 & 0.77697 & 0.17045 \\
1.3 & 0.60427 & 0.74628 & 0.14201 \\
1.4 & 0.59858 & 0.71514 & 0.11656 \\
1.5 & 0.5903 & 0.68373 & 0.09343 \\
1.6 & 0.5801 & 0.65215 & 0.07205 \\
1.7 & 0.56852 & 0.62048 & 0.05196 \\
1.8 & 0.55602 & 0.58874 & 0.03272 \\
1.9 & 0.54293 & 0.55697 & 0.01404 \\
2 & 0.52952 & 0.52517 & 0.00435 \\
\hline
\end{tabular}

ations.

\section{Conclusions}

The main concern of this paper was to construct an approximate analytical solution for the Modified Equations of Emden type. We have achieved this goal by applying DTM. The advantage of DTM is the fact that it provides its user with an analytical approximation. The results confirm that the proposed differential transform method is an effective means of solving various linear and nonlinear differential equations. They also indicate that a small number of arguments are sufficient to provide an accurate solution in practice. An implication of this is that, only a limited number of terms are required to be summed, and therefore the solution may be computed rapidly. However the method gives more satisfactory results for small times which are evident from Figure 1 and we may get better results by adding more terms on the right hand side of Equation (30) for longer time intervals.

\section{References}

[1] V. K. Chandrasekar, M. Senthilvelan and M. Lakshmanan, "On the General Solution for the Modified Emden-Type Equation $\ddot{x}+\alpha x \dot{x}+\beta x^{3}=0$," Journal of Phys- 
ics A, Vol. 40, No. 18, 2004, pp. 4717-4727. doi:10.1088/1751-8113/40/18/003

[2] P. Painleve, "Sur les Equations Différentielles du Second Ordre et d'Ordre Supérieure Dont L'Intégrale Générale est Uniforme,"Acta Mathematica, Vol. 25, No. 1, 1902, pp. 1-85.

[3] E. L. Ince, "Ordinary Differential Equations," Dover, New York, 1956.

[4] H. T. Davis, "Introduction to Nonlinear Differential and Integral Equations," Dover, New York, 1962.

[5] E. Kamke, "Differential Gleichungen Losungsmethoden und Losungen," Teubner, Stuggart, 1983.

[6] G. M. Murphy, "Ordinary Differential Equations and Their Solutions," Van Nostrand, New York, 1960.

[7] V. V. Golubev, "Lectures on Analytical Theory of Differential Equations," Gostekhizdat, Moscow, 1950.

[8] J.S. R.Chisholm and A. K. Common, "A Class Of Second-Order Differential Equations and Related First-Order Systems," Journal of Physics A, Vol. 20, No. 16, 1987, pp. 5459-5472._doi:10.1088/0305-4470/20/16/020

[9] I. C. Moreira, "Lie Symmetries for the Reduced ThreeWave," Hadronic Journal, Vol. 7, 1984, p. 475.

[10] P. G. L. Leach, "First Integrals for the Modified Emden Equation $\ddot{q}+\alpha(t) \dot{q}+q^{n}=0$," Journal of Physics, Vol. 26, No. 10, 1985, p. 2510.

[11] S. Chandrasekhar, "An Introduction to the Study of Stellar Structure,” Dover, New York, 1957.

[12] J. M. Dixon and J. A. Tuszynski, "Solutions of a Generalized Emden Equation and Their Physical Significance," Physical Review A, Vol. 41, No. 8, 1990, pp. 4166-4173. doi:10.1103/PhysRevA.41.4166

[13] G. C. McVittie, "The Mass-Particle in an Expanding Universe," Monthly Notices of the Royal Astronomical Society, Vol. 93, 1933, pp. 325-339.

[14] V. J. Erwin, W. F.Ames and E.Adams, "Wave Phenomenon: Modern Theory and Applications," In: C. Rogers and J. B. Moodie, Eds., Wave Phenomenon: Modern Theory and Applications, North-Holland, Amsterdam, 1984.

[15] F. M. Mahomed and P. G. L. Leach, "The Lie Algebra SL $(3, \mathrm{R})$ and Linearization," Quaestiones Mathematicae, Vol. 12, No. 2, 1989, pp.121-139.

[16] L. G. S.Duarte, S. E. S.Duarte and I. C. Moreira, "One Dimensional Equations with the Maximum Number of Symmetry Generators," Journal of Physics A: Mathematical General, Vol. 20, No. 11, 1987, p. L701. doi:10.1088/0305-4470/20/11/005

[17] S. E. Bouquet, M. R. Feix and P. G. L. Leach, "Properties of Second Order Ordinary Differential Equations Invariant under Time Translation and Self Similar Transformation," Journal of Mathematical Physics, Vol. 32, No. 6, 1991, pp.1480-1490. doi:10.1063/1.529306

[18] W. Sarlet, F. M. Mahomed and P. G. L. Leach, "Symmetries of Nonlinear Differential Equations and Linearization," Journal of Physics A: Mathematical General, Vol. 20, No. 2, 1987, pp.277-292. doi:10.1088/0305-4470/20/2/014

[19] P. G. L. Leach, M. R. Feix and S. Bouquet, "Analysis and Solution of a Nonlinear Second-Order Equation through Rescaling and through a Dynamical Point of View," Journal of Mathematical Physics, Vol. 29, No. 12, 1988, pp. 2563-2569.

[20] R. L. Lemmer and P. G. L. Leach, "The Painlev'e Test, Hidden Symmetries and the Equation $y^{\prime \prime}+y y^{\prime}+k y^{3}=0$," Journal of Physics A: Mathematical General, Vol. 26, 1993. pp. 5017-5024. doi:10.1088/0305-4470/26/19/030

[21] W.H. Steeb, "Invertible Point Transformations and Nonlinear Differential Equations" World Scientific, London, 1993.

[22] M. R. Feix, C. Geronimi, L. Cairo, P.G.L. Leach, R.L. Lemmer and S. Bouquet, "On the Singularity Analysis of Ordinary Differential Equation Invariant under Time Translation and Rescaling," Journal of Physics A: Mathematical General, Vol. 30, 1997, pp. 7437-7461. doi:10.1088/0305-4470/30/21/017

[23] N. H. Ibragimov, "Elementary Lie Group Analysis and Ordinary Differential Equations," John Wiley \& Sons, New York, 1999.

[24] P. G. L. Leach, S. Cotsakis and G. P. Flessas, "Symmetries, Singularities and Integrability in Complex Dynamics II: Rescalings and Time-Translations in 2D Systems," Journal of Mathematical Analysis and Applications, Vol. 251, 2000, pp. 587-608. doi:10.1006/jmaa.2000.7033

[25] V. K. Chandrasekar, M. Senthilvelan and M. Lakshmanan, "On the Complete Integrability and Linearization of Certain Second Order Nonlinear Ordinary Differential Equations," Proceedings of the Royal Society, Vol. 461, No. 2060, 2005, p. 2451. doi:10.1098/rspa.2005.1465

[26] V. K. Chandrasekar, S. N. Pandey, M. Senthilvelan and M. Lakshmanan, "A Simple and Unified Approach to Identify Integrable Nonlinear Oscillators and Systems," Journal of Mathematical Physics, Vol. 47, No. 2, 2006, p.023508.

[27] J. K. Zhou, "Differential Transformation and Its Application in Electrical Circuits," Huazhong University Press, Wuhan, 1986.

[28] S. Mukherjee, B. Roy and S. Dutta, "Solution of DuffingVan der Pol Oscillator Equation by a Differential Transform Method," Physica Scripta , Vol. 83, No. 1, 2010, Article ID 015006. doi:10.1088/0031-8949/83/01/015006

[29] V. K. Chandrasekar, M. Senthilvelan and M. Lakshmanan, "New Aspects of Integrability of Force- Free DuffingVan der Pol Oscillator and Related Nonlinear System," Journal of Physics A, Vol. 37, No. 16, 2004, p. 4527. doi:10.1088/0305-4470/37/16/004

[30] M. Euler, N. Euler and P. G. L. Leach, "The Riccati and Ermakov-Pinney Hierarchies," Report No. 8, Institut Mittag-Leffler, Sweden, 2005/2006.

[31] M. Abramowitz and I. A. Stegun, "Handbook of Mathematical Functions with Formulas, Graphs, and Mathematical Tables," Dover, New York, 1972. 